\title{
Establishment of a population pharmacokinetics model of vancomycin in 94 infants with septicemia and its application in individualized therapy
}

Zhiling $\mathrm{Li}^{1 \dagger}$, Hongjing $\mathrm{Li}^{1 \dagger}$, Chenyu Wang ${ }^{2 \dagger}$, Zheng Jiao ${ }^{2^{*}}$, Feng $\mathrm{Xu}^{3^{*}}$ and Huajun Sun ${ }^{1^{*}}$

\begin{abstract}
Background: We aim to develop a population pharmacokinetics (PopPK) model of vancomycin for the treatment of septicemia in infants younger than one year. Factors influence of the PK was investigated to optimize vancomycin dosing regimen.

Methods: The nonlinear mixed effects modelling software (NONMEM) was used to develop the PopPK model of vancomycin. The stability and predictive ability of the final model were assessed by using normalized prediction distribution errors (NPDE) and bootstrap methods. The final model was subjected to Monte Carlo simulation in order to determine the optimal dose.

Results: A total of 205 trough and peak concentrations in 94 infants (0-1 year of age) with septicemia were analyzed. The interindividual variability of the PK parameter was described by the exponential model. Residual error was better described by the proportional model than the mixed proportional and addition models. Serum creatinine concentration and body weight are the major factors that affect the PK parameters of vancomycin. The clearance was shown to be higher when ceftriaxone was co-treated. More than two model evaluation methods showed better stability than the base model, with superior predictive performance, which can develop individualized dosing regimens for clinical reference. Through prediction of final model, the trough concentration was more likely $<5 \mathrm{mg} / \mathrm{L}$ when a routine dose of $10 \mathrm{mg} / \mathrm{kg}$ is administered every $6 \mathrm{~h}$ to 3-9-month-old infants. Therefore, the dose should be increased in the treatment of infant septicemia.

Conclusions: The stable and effective PopPK model of vancomycin in Chinese infants with septicemia was established. This model has satisfactory predictive ability for clinically individualized dosing regimens in this vulnerable population.
\end{abstract}

Keywords: Infants septicemia, Vancomycin, Population pharmacokinetic, Monte Carlo, Individualized administration

\footnotetext{
* Correspondence: jiaozhen@online.sh.cn; andrewfxu@sina.com; shsetyy@163.com

${ }^{+}$Zhiling Li, Hongjing Li and Chenyu Wang are co-first author and contributed equally to the study.

${ }^{2}$ Department of Pharmacy, Shanghai Chest Hospital, Shanghai Jiao Tong

University, Shanghai, China

${ }^{3}$ Fengxian Hospital, Southern Medical University, Shanghai, China

'Department of Pharmacy, Shanghai Children's Hospital, Shanghai Jiao Tong

University, No. 355 Luding Road, Putuo District, Shanghai 200062, China
}

(C) The Author(s). 2021 Open Access This article is licensed under a Creative Commons Attribution 4.0 International License, which permits use, sharing, adaptation, distribution and reproduction in any medium or format, as long as you give appropriate credit to the original author(s) and the source, provide a link to the Creative Commons licence, and indicate if changes were made. The images or other third party material in this article are included in the article's Creative Commons licence, unless indicated otherwise in a credit line to the material. If material is not included in the article's Creative Commons licence and your intended use is not permitted by statutory regulation or exceeds the permitted use, you will need to obtain permission directly from the copyright holder. To view a copy of this licence, visit http://creativecommons.org/licenses/by/4.0/ The Creative Commons Public Domain Dedication waiver (http://creativecommons.org/publicdomain/zero/1.0/) applies to the data made available in this article, unless otherwise stated in a credit line to the data. 


\section{Background}

Over the last decades, the most frequent causative agents of septicemia in neonates have been reported to be the Gram-positive cocci infections, especially methicillin-resistant Staphylococcus aureus (MRSA) [1]. Vancomycin is widely used in clinical scenarios as the antimicrobial therapy for targeted or empiric treatment of neonatal sepsis [2]. It is the first glycopeptide antibiotic with triple mechanisms, inclusive of inhibiting the synthesis of cell walls and RNA in the cytoplasm, and altering membrane permeability in bacteria [3]. However, there remain numerous disputes regarding the current individualization of vancomycin dose in clinical practice. Even with the routine dose of vancomycin in newborns admitted in the intensive care unit, we are still unable to easily obtain trough and peak plasma concentrations of 5 to $10 \mathrm{mg} /$ liter and 20 to $50 \mathrm{mg} /$ liter in neonate patients [4].

Vancomycin-associated nephrotoxicity is a critical appraisal of risk with high-dose therapy for infants and adults [5]. Nevertheless, the immaturity of physiological processes and organ functions, such as liver's and kidney's, predisposes infants to disparate drug disposition and responses compared with that in adults [5, 6]. It is especially important to avoid exposure to unnecessarily high peak or trough concentrations of vancomycin in the premature newborn. The pharmacokinetic (PK) and pharmacodynamic (PD) parameters constantly change across the pediatric groups. The disposition and response of infants are not only different from that of other aged children but also widely vary within themselves $[6,7]$. These issues along with the lack of pharmacokinetics data on infants lead to remarkable difficulty in establishing the regimen for infants. Based on the assessment of population PK models, population PK parameter estimates are less biased than those obtained using the naive and standard two-stage approaches. Thus, the NONMEM software is the most widely used software for the characterization of population PK in clinical practice in order to solve the problem [8]. To date, vancomycin has been one of the most studied antibiotics using the PopPK in neonates and adults [9]. Approaches to vancomycin clinical dosing were developed on basis of the PK parameters of individuals [10]. The method does not only conduct statistical analysis using the sparse uneven data for each patient with a few samplings, but also integrate gestational age, gestational weeks, weight, and other important data of infants for PK data processing, which suitable for infants [10, 11]. Our previous investigation characterized the PK parameters of neonates [12], but the optimal use of vancomycin in infants need to be conducted continually. It is challenging to develop and validate a model that prospectively predicts individual exposure in infants and further optimize dosing regimens. Vancomycin is always used to combine with other antibiotics such as ceftriaxone and meropenem. In order to know whether drug interactions influence the blood concentration of vancomycin, this study aims to identify individual factors that affect drug interactions of ceftriaxone that subsequently influences variability and to establish dosing regimens for infants in China.

In this study, the PK parameters that affect the PK of vancomycin were completely elucidated, and the PopPK of vancomycin in infants (0-1 year of age) with septicemia in a Chinese population was developed. Based on the PopPK model, we screened for factors that might significantly influence the PK of vancomycin and provide a reference for the individualization of treatment with vancomycin in clinical practice. Our work would potentially reduce the incidence of adverse reactions originated from the use of vancomycin and improve efficacy and safety.

\section{Methods}

\section{Patients and data collection}

A total of 94 Chinese infants who received vancomycin for the treatment of septicemia at a grade A special hospital between January 2009 and December 2015 were enrolled. The following data were collected: blood concentration of vancomycin, daily vancomycin dose, demographic characteristics of patients, blood examination results, hepatic and renal function data, and coadministered medicines. Serum vancomycin concentration was determined by chemiluminescence analysis.

Table 1 Demographic and Clinical Data of Infants

\begin{tabular}{lll}
\hline Characteristics & Mean \pm SD & Median (range) \\
\hline The number of infants (M/F) & $94(58 / 36)$ & \\
Samples & 205 & \\
Age (d) & $67.14 \pm 80.85$ & $88.5(1-345)$ \\
Weight (kg) & $4.686 \pm 2.57$ & $4(1.4-18)$ \\
Height (cm) & $54.29 \pm 8.28$ & $52(37-78)$ \\
Gestational age (week) & $37.18 \pm 3.71$ & $39(25.7-41.4)$ \\
Correct gestational age (week) & 46.44 & $43(31.14-83.07)$ \\
Birth weight (g) & $2978 \pm 815.49$ & $3200(850-4400)$ \\
Creatinine levels ( $\mu$ mol/L) & $19.91 \pm 7.45$ & $18.25(5.5-50)$ \\
Creatinine clearance (ml/min/1.73m²) & $111.1 \pm 49.71$ & $120(21.46-280)$ \\
Daily dose (mg/day) & $74.69 \pm 44.95$ & $60(20-200)$ \\
Observed concentration (ug/ml) & $13.35 \pm 10.82$ & $10.6(3.31-51.93)$ \\
ALT (U/L) & $29.63 \pm 27.14$ & $20(3-156)$ \\
AST (U/L) & $48.2 \pm 35.82$ & $34.5(13-241)$ \\
BUN (mmol/L) & $3.11 \pm 1.83$ & $2.75(0.6-9.5)$ \\
The total protein (g/L) & $52.59 \pm 7.95$ & $53(32.4-78)$ \\
Albumin (g/L) & $34.23 \pm 5.37$ & $35(18.45-43)$ \\
\hline
\end{tabular}


Demographic and clinical data of infants are summarized and presented in Table 1.

Using the fluorescence polarization immunoassay method with the AxSYM system, serum vancomycin concentrations were measured as part of the therapeutic drug monitoring activity in the hospital. The assay sensitivity limit was $2.00 \mathrm{mg} / \mathrm{L}$. Coefficients of variation were $4.26,2.94$, and $4.06 \%$ at concentrations of $7.0,35.0$, and $75.0 \mathrm{mg} / \mathrm{L}$, respectively (package insert, Axsym system, Abbot Laboratories, AbbotPark, IL, USA).

\section{PopPK modeling}

The PopPK model was developed using the NONMEM version 7.2 (Icon Inc., PA, the USA), Fortran compiler: Intel Fortran XE 2011 Update 13 (Intel Corp, CA, the USA),Wings for NONMEM (version 7.2, Nick Holford, University of Auckland, Auckland, New Zealand, http:// wfn.sourceforge.net), Xpose (version 4.2.1, Department of Pharmaceutical Biosciences at Uppsala University), $\mathrm{R}$ package (version 2.15.1, http://www.r-project.org), Perlspeaks-NONMEM version 3.5.3 (http://psn.sf.net), Perl (ActivePerl-5.14.2, 64 bit), and Pirana (version 2.7.0, http://www.pirana-software.com). NONMEM was used to employed pharmacokinetic analysis. Based on individual dataset, population values of the PK parameters were considered as fixed-effect parameters. Inter- and intraindividual variabilities were estimated as random effects.

205 trough and peak about vancomycin concentrations versus time were fitted to both one- and twocompartmental models with first-order elimination. The most suitable compartmental model was determined to be specified to NONMEM by ADVAN1-TRANS2 or ADVAN3-TRANS4 subroutines. By using these model specifications, the fixed-effect PK parameters were directly estimated. Total body clearance $(\mathrm{CL})$ and volume of distribution (V) are for the one-compartmental model, while $\mathrm{CL}$, volume of distribution of the central compartment $\left(\mathrm{V}_{1}\right)$, intercompartment clearance $(\mathrm{Q})$, and volume of distribution of the peripheral compartment $\left(\mathrm{V}_{2}\right)$ for the two-compartmental model. Additive, proportional, and exponential error models were tested to describe inter- and intraindividual variabilities.

In preliminary screening phase, each of covariates that significantly improved the predictive ability of the basic model would be included. Observed concentration-time profile was used to compare. The influence of covariates on the PK parameters of vancomycin as follows: weight, age, sex, serum creatinine (SCR) concentration, blood urea nitrogen, creatinine clearance rate [CLCR, using the Modification of Diet in Renal Disease/MDRD4 equation [10] and Cockcroft-Gault (C-G) equation [13]], serum albumin concentration, aspartate transaminase and alanine transaminase levels, and concomitant drugs (ceftriaxone, meropenem, gentamicin, furosemide, ibuprofen, and dexamethasone). The significant covariates were then cumulatively added (forward stepwise fashion) to the model in the order of their contribution in reducing the objective function value (OFV, $-2 \log$ likelihood difference) in the preliminary analysis until no further reduction in OFV is observed. Finally, back elimination was conducted to eliminate any unnecessary covariate from the full regression model in the descending order of their contribution to the change in OFV.

The best compartmental model (one vs. two compartments), error model (additive vs. proportional vs. combined error models), and the retention of covariate (s) in the model were determined with the statistical significance of the model and were evaluated via the likelihood ratio test using the minimum value of the OFV, as produced by the NONMEM program. Changes in OFV of $>6.63$ and $>9.21$ were considered significant based on the $x^{2}$ distribution with degree of freedom $(\mathrm{df})=1$ and 2 (both $P<0.01$ ), respectively [14].

Other diagnostic criteria were a reduction in the unexplained interindividual variability for the associated PK parameters and an improvement in the graphic diagnostic model. Graphics were obtained using plotted observed vs. predictive concentrations and observed vs. weighted residuals (predicted minus observed concentrations and weighted by standard deviation). First-order conditional estimation interaction was utilized to estimate the PopPK parameters. Covariates were screened according to the stepwise method, and the final PopPK model of vancomycin was then established by backward elimination. The exponential model was used to describe interindividual variability of the PK parameters. Residual error was better described by the exponential model than the mixed exponential and addition models.

\section{Covariate screening}

Before covariate analyses, any correlation between the covariates (correlation coefficient $>0.5$ ) should be examined, and the study should only choose one for the analysis. Using the Xpose 4 program package of the $R$ language to draw each co-distribution map, it was examined whether the distribution of data is normal [15]. Parameter values of the final model is shown in Table 2 and screening process was summarized in Table 3.

\section{Model evaluation}

Internal evaluation is the method in which original modeling data are used in resampling technology and in the establishment of a validation dataset to validate the model. The normalized prediction distribution errors (NPDE), and bootstrap methods were used to test the model [16]. The results of boostrap in final model were summarized in Table 4 . The stability and predictive ability of the final model were evaluated using NPDE, 
Table 2 Parameter Value of the Final Model

\begin{tabular}{lllll}
\hline Parameter & Definition & Estimates & RSE (\%) & 95\% confidence interval \\
\hline $\mathrm{CL}$ & clearance & 10.3 & $29.60 \%$ & $4.322-16.278$ \\
$V$ & distribution volume & 50.6 & $7.50 \%$ & $43.211-57.989$ \\
$\Theta 1$ & weight coefficient on CL & 1.06 & $9.40 \%$ & $0.865-1.255$ \\
$\Theta 2$ & Serum creatinine coefficient on CL & -0.315 & $20.70 \%$ & $-0.443--0.187$ \\
$\Theta 3$ & Co-therapy with ceftriaxone coefficient on CL & 1.46 & $16.70 \%$ & $0.982-1.938$ \\
$\eta 1$ & Between-subject variability of Clearance & 0.145 & $27.70 \%$ & \\
$\varepsilon 1$ & Proportional within-subject variability & 0.194 & $16.10 \%$ & \\
\hline
\end{tabular}

bootstrap method, and external data. The results of the model average prediction error (MPE) and average absolute error (MAE) calculated by the external evaluation method are shown in Table 5.

\section{Monte Carlo simulation}

The final model was subjected to Monte Carlo simulation to determine the optimal dose under which the drug concentration of a certain patient can be controlled within the reference concentration. According to the range of the commonly used dose in infants and factors that set the scene, data files of each scene were written. Based on the PopPK model of vancomycin, data files of each scenario were simulated using the Monte Carlo method, and data of 5000 were generated, which were completed by the ONLYSIMULATION and SUBPROBLEMS modules in the NONMEM software SIMULATION.

\section{Results}

Basic model

This study established the PopPK model according to results of the conventional therapeutic drug monitoring (TDM), which only had the fixed sampling point trough and peak concentrations. Data on vancomycin concentrations versus time were fitted to both one- and twocompartmental models, respectively. Because sparse sampling method, there is not available data enough to establish a two-compartmental (Table 3). The results preferred the one-compartmental model as more suitable model to describe this set of data.

The additive, proportional, and exponential error models were tested to describe inter- and intraindividual variabilities. Interindividual variability was estimated using an exponential error model. Because of the extremely large relative standard deviation in the interindividual variation of $\mathrm{V}$, which was not allowed, the ETA test was not conducted. Bootstrap is used to estimate statistical variance and interval estimation of the statistical method, which could be used to estimate the confidence interval of parameter values of $2.5-97.5 \%$. If parameter values of the $95 \%$ confidence interval include 0 , then the parameter estimates are not reliable.

For the residual variance model, the mixed model of proportional and additive errors was examined, and the proportional residual model is adopted.

Table 3 Model Selection Process ${ }^{a}$

\begin{tabular}{lllll}
\hline Model & Description \& main characteristics & OFV value & $\Delta$ OFV value & Whether or not included \\
\hline 1 & One compartment model & 1098.753 & 0 & YES \\
2 & One compartment model, ETA was not estimated on V & 1102.375 & 3.622 & NO \\
Forward inclusion process & & & YES \\
3 & Add WT on CL & 1000.624 & -69.045 & YES \\
4 & Add SCR on CL & 988.23 & -12.394 & YES \\
5 & Add co-therapy with ceftriaxone on CL & 978.401 & -9.829 & YES \\
Backward elimination process & & & YES \\
6 & Remove WT on CL & 1016.409 & 44.627 & 14.602 \\
8 & Remove SCR on CL & 987.288 & 8.187 & YES \\
\hline
\end{tabular}

${ }^{a}$ This is a standard stepwise procedure for screening covariates in popPK analysis 
Table 4 Bootstrap Results of Final Model

\begin{tabular}{lllllllll}
\hline Percentiles & OFV & CL & V & WT & SCR & DC & $\begin{array}{l}\text { BSV } \\
\text { CL }\end{array}$ & ERR1 \\
\hline medians (50\%) & 964.99 & 11.01 & 50.38 & 1.06 & -0.34 & 1.40 & 0.14 & 0.19 \\
$0.50 \%$ & 811.46 & 5.17 & 40.37 & 0.82 & -0.66 & 0.89 & 0.03 & 0.11 \\
$2.50 \%$ & 844.48 & 5.93 & 43.07 & 0.87 & -0.54 & 0.97 & 0.05 & 0.13 \\
$5 \%$ & 863.64 & 6.51 & 44.41 & 0.90 & -0.49 & 1.01 & 0.06 & 0.14 \\
$95 \%$ & 1079.97 & 17.96 & 56.70 & 1.25 & -0.22 & 1.75 & 0.21 & 0.25 \\
$97.50 \%$ & 1100.29 & 22.17 & 57.74 & 1.31 & -0.20 & 1.79 & 0.23 & 0.27 \\
$99.50 \%$ & 1149.60 & 28.42 & 60.35 & 1.40 & -0.08 & 1.91 & 0.26 & 0.31 \\
\hline
\end{tabular}

\section{Covariate Models}

As depicted in Fig. 1, data obtained in this study had significant differences between the newborns and infants. The distribution of some covariates was not normal, including body weight, age, and SCR. Because the relationship between the clearance rate and body weight was obviously different at different phages, the method of subsection and different velocities should be adopted. Because the OFV value after adding weight generally decreased by $>100$, body weight was considered an important covariate that influences the clearance rate and should be retained. Results of the second method showed that the correction of the prediction error was $>50 \%$, which did not meet the requirements, and results of the first method had good stability. Thus, model 2 should be chosen for the next step of modeling.

First, based on the first method model, SCR concentration and CLCR were added to the CL as an impact factor; then, the two covariates were compared to identify the one that could better describe the CL. Results showed that OFV values decrease after the addition of SCR concentration and CLCR, and the OFV of the multiplication method would decrease more than the addition method. Because the OFV of the two models were similar and creatinine value was more convenient to obtain, which is much useful in future clinical development, the model for SCR as the covariate should be chosen.

Table 5 Prediction Ability of the Basic and Final Models

\begin{tabular}{lll}
\hline Evaluation & Final model & Basic model \\
\hline MPE (\%) & 21.306 & 43.116 \\
SPE (\%) & 63.627 & 101.701 \\
MAE (\%) & 54.825 & 74.428 \\
RMSE (\%) & 65.647 & 108.211 \\
MDPE (\%) & 14.451 & 33.177 \\
MDAE (\%) & 46.361 & 48.575 \\
MBA (\%) & 4.498 & 10.195 \\
SDBA (\%) & 55.400 & 70.967 \\
\hline
\end{tabular}

The effects of concomitant drugs on clearance were investigated. The OFV of meropenem and ceftriaxone covariates decreased by $>3.84$. Thus, meropenem and ceftriaxone should both be incorporated. The OFV of meropenem elimination decreased $<6.635(P>0.01)$, which was not statistically significant. Meanwhile, combination with meropenem neither had an effect on CL $(<20 \%)$ nor had clinical significance. Thus, meropenem should be removed and other covariates should be retained.

\section{Final model}

The final regression model was as follows:

$$
\begin{aligned}
C L\left(\frac{L}{h r}\right)= & 10.3 \times\left(\frac{\mathrm{WT}(\mathrm{kg})}{70}\right)^{1.06} \\
& \times\left(\frac{\mathrm{SCR}(\mu \mathrm{mol} / \mathrm{L})}{20}\right)^{-0.315} \times 1.46^{D C} \\
V(L)= & 50.6 \times\left(\frac{\mathrm{WT}(\mathrm{kg})}{70}\right)
\end{aligned}
$$

$\mathrm{DC}=1$ when incorporated with ceftriaxone, then $\mathrm{DC}=$ 0.

SCR and body weight were the major factors influencing the PK parameters of vancomycin. The clearance would be larger when ceftriaxone is incorporated.

Compared with the basic model, the external validation of the final model showed that the fitting degree of the predicted and measured values obviously improved, and the individual value average prediction errors of the final model was smaller but higher in the forecast precision. Then, the final model showed better fitting effect after adding the fixed-effect factors (Table 2).

\section{Model evaluation}

In order to calculate the predictive error of each sampling point, we made MPE and MAE diagram for the basic model and the final model. It can be seen from Table 5 that the inter-individual variation of the pharmacokinetic parameters in the final vancomycin PopPK model is significantly reduced compared with the basic model. The NPDE was used to validate the prediction performance of the model. The NPDE was homogeneity of variance and normal distribution according to the quantile plots (Fig. 2), NPDE distribution, and statistical test results. The $t$-test result was 0.566, and the Fisher's variance test result was 0.859 . Such results indicated that the NPDE-preferred model can be used to generate analog data.

At the same time, 1000 data were created for bootstrap validation based on a non-parametric method to obtain 1000 set of model parameters for statistical calculations. If the parameter value was not significantly different, then the model was stable. In this study, more 


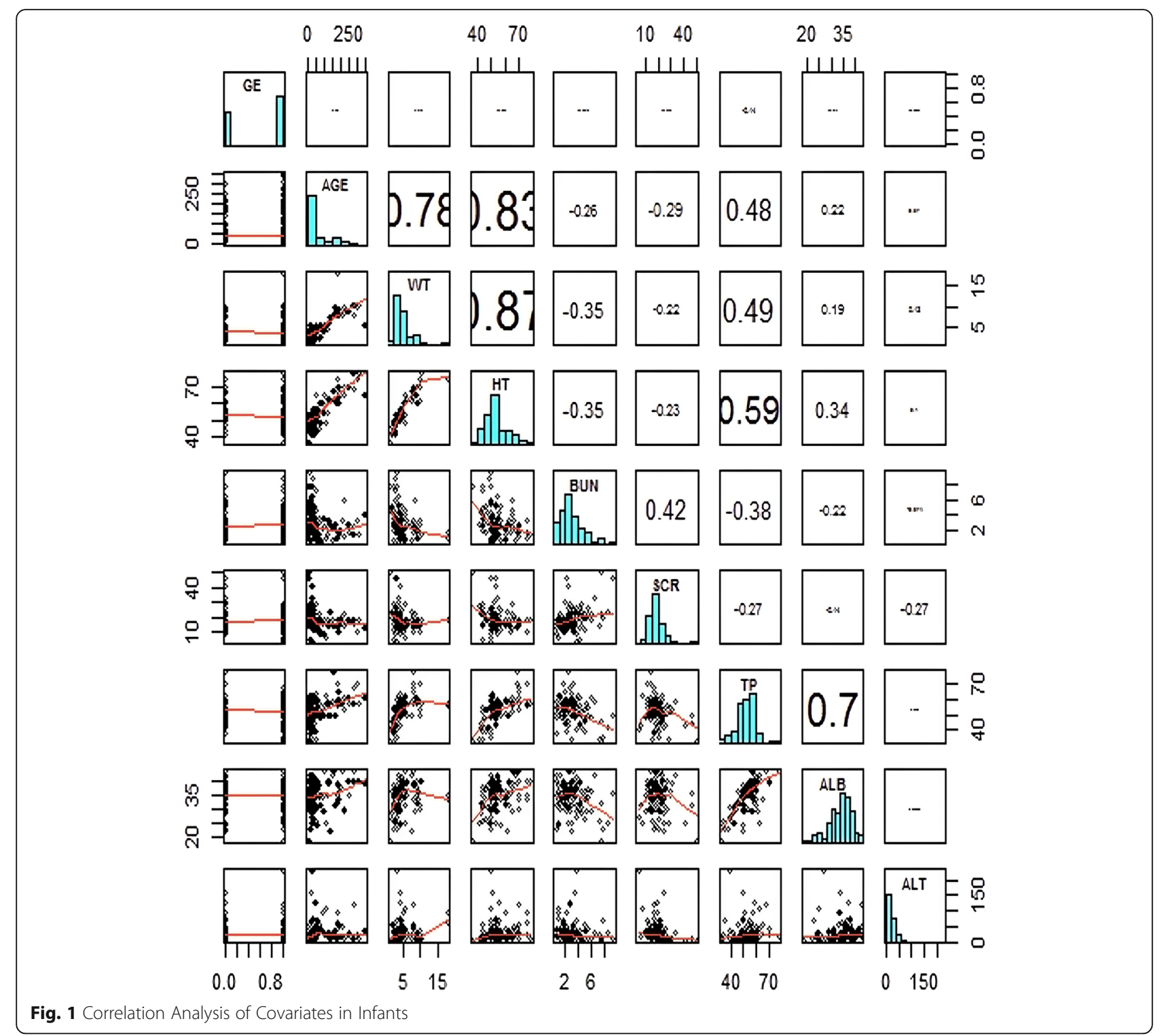

than two model validation methods showed that the final internal model had good stability with superior predictive performance, which can be used to develop individualized dosing regimens for clinical reference.

The performance of the final covariate model was evaluated by visual inspection of diagnostic scatter plots. The robustness of the model was assessed using a nonparametric bootstrap, with replacement, of 1000 NONMEM runs of the final model; the bootstrap median parameter values and the percentile bootstrap 95\% intervals were compared with the respective values estimated from the final model. The final model's 25 estimates were terminated. In addition, the 2.5 to $97.5 \%$ confidence interval of $\mathrm{CL}, \mathrm{V}$ and the estimated results of the covariable parameters were not covered to 0 , which met the requirements. All of results are summarized in Table 4.

\section{Monte Carlo simulation}

The steady state of infants administered with vancomycin was designed, and an analysis was conducted using Monte Carlo simulation to generate simulated data. In children with infection who were taking vancomycin at a steady state, the Monte Carlo simulation generated analog data analysis results showing that trough concentrations of the dosing regimen $(15 \mathrm{mg} / \mathrm{kg})$ every $24 \mathrm{~h}$ in infants who are 29 weeks premature would be < $5 \mathrm{mg} / \mathrm{L}$, and this did not even meet the dose requirement of $10-15 \mathrm{mg} / \mathrm{L}$ [4]. The dosing interval should be shortened; although trough concentrations in 28-day-old neonates were only $4.3 \mathrm{mg} / \mathrm{L}$, the peak concentrations were up to $72 \mathrm{mg} / \mathrm{L}$. Trough concentrations are more likely to be $<5 \mathrm{mg} / \mathrm{L}$ when a dose of $10 \mathrm{mg} / \mathrm{kg}$ is administered every $6 \mathrm{~h}$ in 3-9-month-old infants; thus, the dose 
Q-Q plot versus N(0,1)for npde

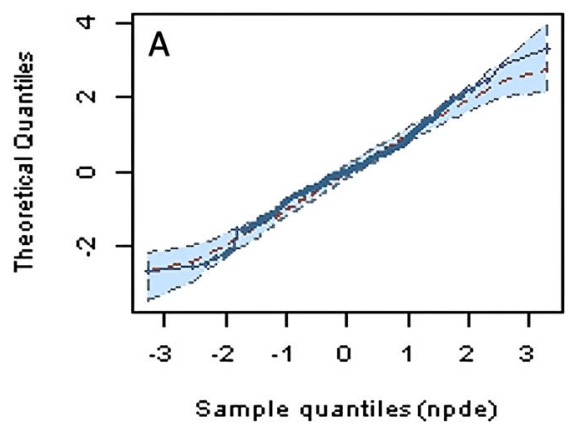

C

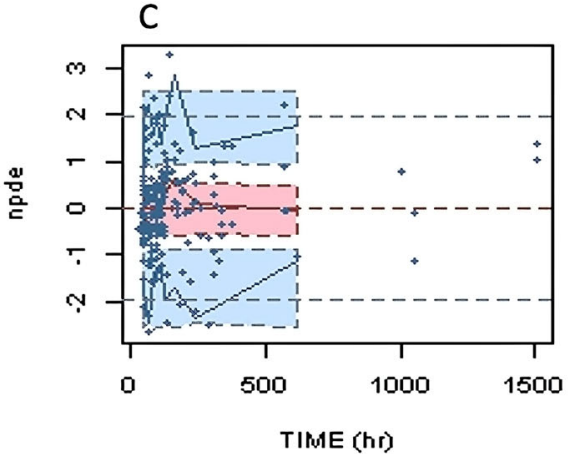

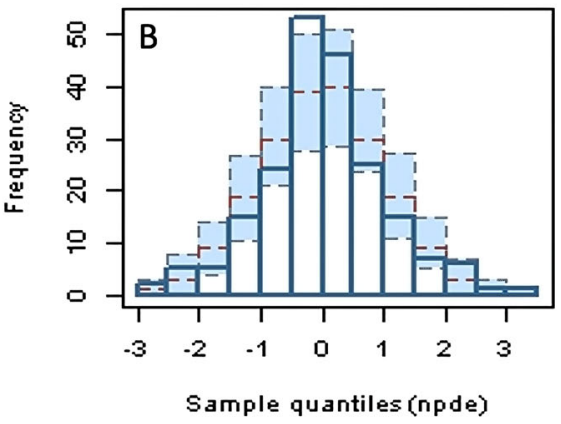

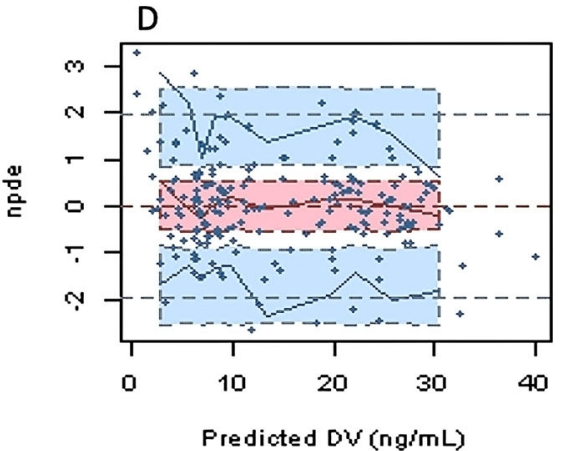

Fig. 2 Normalized prediction distribution error (NPDE) for the final model. a Quantile-quantile plots of NPDE vs. the expected standard normal distribution; b Histogram of NPDE values with the standard normal distribution overlay; c Scatter plot of the time vs. NPDE; d. Scatterplot of predictions vs. NPDE

should be increased. The clinical application mode of vancomycin has been established and used in individual drug administration in clinical practice, as shown in Fig. 3.

\section{Discussion}

The PK of vancomycin in compartmental model studies, including one-, two-, and three-compartmental models, is controversial. In recent years, one-compartmental model has been successfully used in the study of neonates [17], children [18], adults [19], and different disease statuses of patients [20], which can better meet the needs of the fitting model and estimation of the PK parameters. Because of limited information on drugs, the results preferred two-compartment model as better model to describe the present population. In this study, the PopPK model was successfully established using the one-compartment model as the basis of the structural model for PopPK analysis.

The PopPK model can fully consider the intra- and inter-individual variability; thus, it is advantageous for individual drug delivery. The mixture model was fitted with the simultaneous presence of proportional and additive errors. Factors that influence the PK of vancomycin were selected and investigated, and the effects of weight, SCR, and combined use of drugs were investigated. When screening weight, two methods were used, and meropenem and ceftriaxone as concomitant drugs were also assessed.

Most of the vancomycin in the body were eliminated by the prototype, and the effect of renal function [CLCR or SCR concentrations] on vancomycin clearance rate was investigated. The CLCR data were estimated using the Schwartz formula, and the final model indicated that SCR was an important factor affecting the vancomycin clearance rate. Moreover, the results were consistent with the those reported by Reveilla [21] and Llopis-Salvi [22]. This study first used LZL12B1 as the basic model, adding SCR concentration and CLCR as factors influencing $\mathrm{CL}$, and compared the two covariates to determine which among the two can better describe the CL. Results showed that the addition of SCR concentration and CLCF after the model OFV decreased, and there was a greater reduction in multiplication and addition after the addition of OFV.

Age, weight, and height had an extremely high linear correlation, and height and TP and TP and ALB also had correlation coefficient of $>0.5$; thus, attention should be paid to the association between these factors, and such factors must not be added at the same time. In addition, after adding weight, the OFV value decreased by $>100$; thus, weight should be retained because it has 

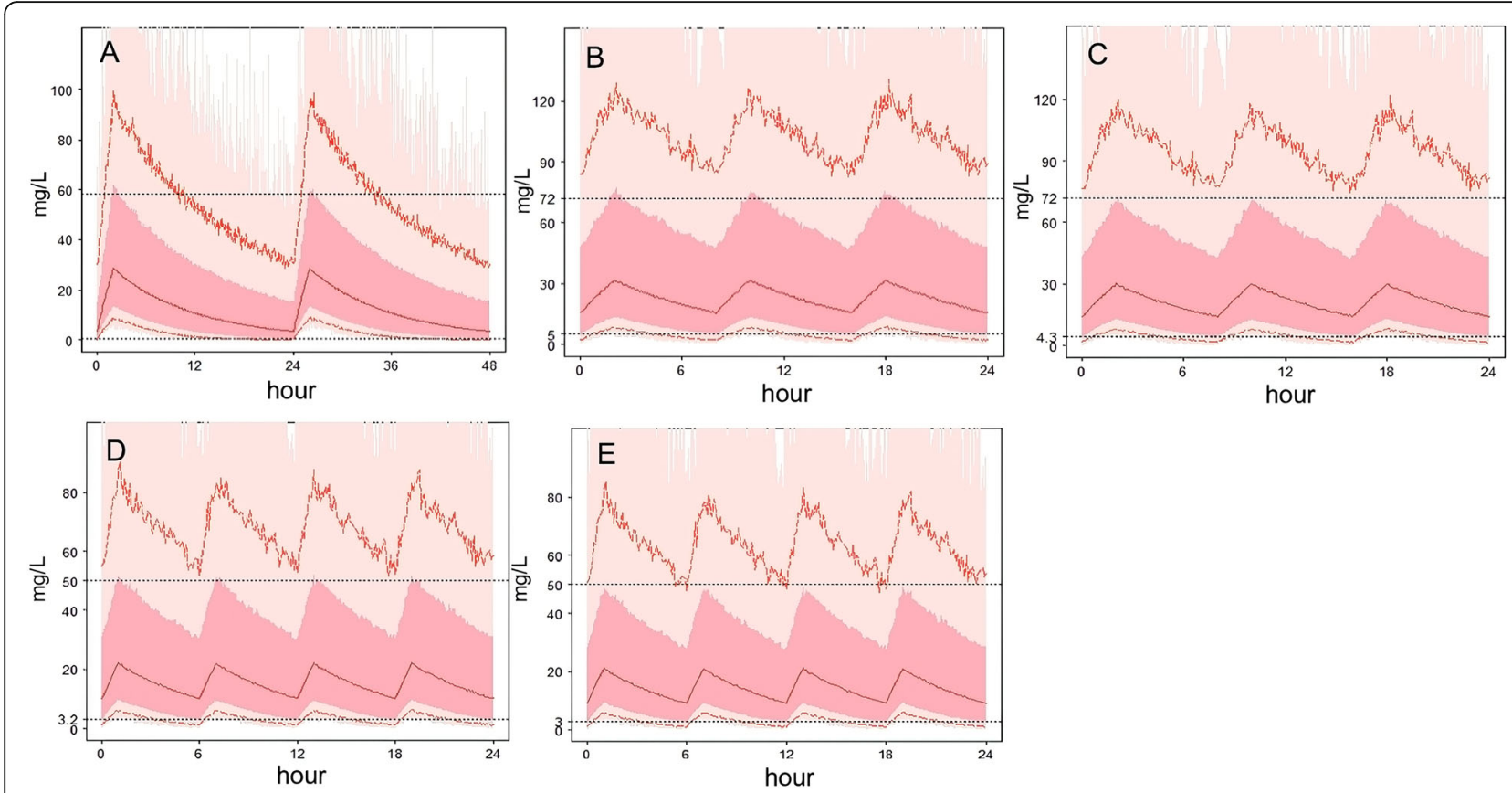

Fig. 3 Distribution of the concentration range of vancomycin. Vancomycin concentration-time profile from the Monte Carlo simulation with five typical cases. Deep and light pink colors represent the 5th and 95th percentiles of the simulated data, respectively. Red solid line represents the lower and upper boundaries of the therapeutic range of vancomycin. Horizontal axis: Time after achieving steady state (h); vertical axis: vancomycin concentration (mg/L). a. Neonates with $0.95 \mathrm{~kg}$ body weight (gestational age: 29 weeks, SCR: 100umol/L, $19 \mathrm{mg}$ every $24 \mathrm{~h}$ ); $\mathbf{b}$.

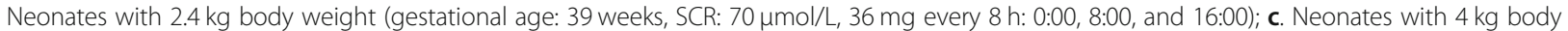
weight (gestational age: 39 weeks, 28 days, SCR: $60 \mu \mathrm{mol} / \mathrm{L}, 60 \mathrm{mg}$ every $8 \mathrm{h:}$ 0:00,8:00, and 16:00); d. Infants with $5 \mathrm{~kg}$ body weight (gestational age: 39 weeks, 3 months, SCR: 32 mmol/L, 50 mg every 6 h: 0:00,6:00,12:00, and 18:00); e. Infants with 8 kg body weight (gestational age: 39 weeks, 9 months, SCR: $28 \mu \mathrm{mol} / \mathrm{L}, 80 \mathrm{mg}$ every $6 \mathrm{h:0}: 00,6: 00,12: 00$, and 18:00)

an important influence on the clearance rate of covariates. Stability and accuracy were in accordance with the requirements.

This study examined the effect on vancomycin elimination rate while administered in combination of other antimicrobial agents (ceftriaxone, meropenem, and gentamicin), furosemide, ibuprofen, and dexamethasone. Based on the basic model, factors of drug combination were investigated, and those that accounted for $>10 \%$ of cases were internalized. The effect of drug combination on the clearance rate was investigated using LZL12WS3, respectively in additional method with meropenem and ceftriaxone covariates. Finally, after drawing the Xpose program [15], the distribution of covariates showed a straight line, indicating that the difference between values of covariates and the final model was not statistically different. Thus, the final model only included ceftriaxone.

Because the renal pathway is primarily involved in vancomycin elimination, it is not surprising that the creatinine level is a significant factor of vancomycin clearance. More that $80 \%$ of vancomycin intravenously administered are excreted via the urine, and the total body clearance rate is related to renal function. Thus, ceftriaxone may competitively inhibit vancomycin. This study showed that the use of ceftriaxone increases vancomycin clearance rate. Therefore, the protein binding rate of ceftriaxone may be high and have competitive inhibition to vancomycin, and the uncombined vancomycin levels increasingly cleared up [23]. Besides, ceftriaxone may affect the glomerular filtration of vancomycin because the organs of infants were not mature. Similar reports have not yet been published, and the detailed mechanism must be explored in future studies.

Based on the recent guidelines, vancomycin trough concentration of $>10 \mathrm{mg} / \mathrm{L}$ was recommended to prevent the development of resistance [24]. Vancomycin trough concentrations of $15-20 \mathrm{mg} / \mathrm{L}$ were used to treat serious infections such as endocarditis, osteomyelitis, meningitis, and hospital acquired pneumonia. Predictive performance in the validation step was evaluated by applying the final model to the validation group. The results of this study can be directly applied in clinical practice, and the model can be used to obtain specific PK parameters of a patient to establish vancomycin dosage regimen in patients similar to those in the present study. 
The PK parameters of vancomycin in infants with septicemia younger than one year in China were established, and factors influencing the variability of these PK parameters were identified. Inter- and intra-individual variabilities of the PK parameters were determined. The established PK model in this study was stable and had good prediction ability, which can be used to obtain specific PK parameters to establish vancomycin dosage regimen in patients similar to those in the present study and to develop individualized clinical dosing.

\section{Conclusions}

The commended vancomycin dose for infants according to the latest guidelines and instructions is more likely not to obtain target trough concentrations $[4,25]$. The median concentration during 29 weeks of gestation was only $3.66 \mathrm{mg} / \mathrm{L}$. We found the dose should be increased and the dosing interval should be decreased by applying final model. As a result, the clinical application of the PopPK model of vancomycin established in the present study can promote the rational use of drugs in clinical practice.

\section{Abbreviations}

PopPK: Population pharmacokinetics; MRSA: Methicillin-resistant Staphylococcus aureus; PK: Pharmacokinetic; PD: Pharmacodynamic; SCR: Serum creatinine; OFV: Objective function value; NPDE: Normalized prediction distribution errors; TDM: Therapeutic drug monitoring; AST: Aspartate aminotransferase; ALT: Alanine aminotransferase; BUN: Blood Urea Nitrogen; TP: Total protein

\section{Acknowledgments}

We would like to express my gratitude to all those who helped me during the writing of this paper. Our deepest gratitude goes first and foremost to Professor Xiaohu Gong for assisting in preparation of this manuscript. Second, we would like to express my heartfelt gratitude to Professor Cheng Cai, Yong Hu and Yihuan Chen, who have instructed and helped me a lot in collecting the patient's information.

\section{Authors' contributions}

ZJ, FX and HJS conceived and designed the study. ZLL, HJL, and CYW collected the information of the neonates. ZLL, HJL, and CYW performed the experiments. ZLL, HJL, and ZJ wrote the paper. ZLL, HJL, and CYW built and evaluated the model. ZJ, FX and HJS reviewed and edited the manuscript. All authors read and approved the manuscript.

\section{Funding}

The study design, data acquisition and language editing of this work was supported by the Wu Jieping Foundation of Clinical Pharmacy Branch of Chinese Medical Association (No. 320.6750.19090-1).

\section{Availability of data and materials}

All data generated or analysed during this study are included in this published article.

\section{Declarations}

Ethics approval and consent to participate

This study was approved by Ethic Committee of Shanghai Children's Hospital (2015R003-F01). Written informed consent was obtained from guardian.

Consent for publication

Not applicable.

\section{Competing interests}

The authors declare that they have no conflict of interest.

Received: 5 August 2020 Accepted: 15 April 2021

Published online: 04 May 2021

\section{References}

1. Kriebs JM. Staphylococcus infections in pregnancy: maternal and neonatal risks. J Perinat Neonatal Nurs. 2016;30(2):115-23. https://doi.org/10.1097/jpn. 0000000000000165.

2. Peixoto PB, Massinhani FH, Netto Dos Santos KR, Chamon RC, Silva RB, Lopes Correa FE, et al. Methicillin-resistant Staphylococcus epidermidis isolates with reduced vancomycin susceptibility from bloodstream infections in a neonatal intensive care unit. J Med Microbiol. 2020;69(1):415. https://doi.org/10.1099/jmm.0.001117.

3. Liu C, Bayer A, Cosgrove SE, Daum RS, Fridkin SK, Gorwitz RJ, et al. Clinical practice guidelines by the infectious diseases society of america for the treatment of methicillin-resistant Staphylococcus aureus infections in adults and children: executive summary. Clin Infect Dis. 2011;52(3):285-92. https:// doi.org/10.1093/cid/cir034.

4. Tobin CM, Darville JM, Thomson AH, Sweeney G, Wilson JF, MacGowan AP, et al. Vancomycin therapeutic drug monitoring: is there a consensus view? The results of a UK National External Quality Assessment Scheme (UK NEQA S) for antibiotic assays questionnaire. J Antimicrob Chemother. 2002;50(5): 713-8. https://doi.org/10.1093/jac/dkf212.

5. Wong-Beringer $A$, Joo J, Tse E, Beringer P. Vancomycin-associated nephrotoxicity: a critical appraisal of risk with high-dose therapy. Int J Antimicrob Agents. 2011;37(2):95-101. https://doi.org/10.1016/j.ijantimicag.2 010.10.013.

6. Gonzalez D, Melloni C, Yogev R, Poindexter BB, Mendley SR, Delmore P, et al. Use of opportunistic clinical data and a population pharmacokinetic model to support dosing of clindamycin for premature infants to adolescents. Clin Pharmacol Ther. 2014;96(4):429-37. https://doi.org/10.1038/ clpt.2014.134.

7. Kimura T, Sunakawa K, Matsuura N, Kubo H, Shimada S, Yago K. Population pharmacokinetics of arbekacin, vancomycin, and panipenem in neonates. Antimicrob Agents Chemother. 2004;48(4):1159-67. https://doi.org/10.112 8/aac.48.4.1159-1167.2004.

8. Ette El, Williams PJ. Population pharmacokinetics II: estimation methods. Ann Pharmacother. 2004;38(11):1907-15. https://doi.org/10.1345/aph.1E259.

9. Spruill WJ, Wade WE, Cobb HH 3rd. Estimating glomerular filtration rate with a modification of diet in renal disease equation: implications for pharmacy. Am J Health Syst Pharm. 2007;64(6):652-60. https://doi.org/1 0.2146/ajhp060239.

10. Brendel K, Dartois C, Comets E, Lemenuel-Diot A, Laveille C, Tranchand B, et al. Are population pharmacokinetic and/or pharmacodynamic models adequately evaluated? A survey of the literature from 2002 to 2004. Clin Pharmacokinet. 2007;46(3):221-34. https://doi.org/10.2165/00003088-20074 6030-00003

11. Maitre PO, Buhrer M, Thomson D, Stanski DR. A three-step approach combining Bayesian regression and NONMEM population analysis: application to midazolam. J Pharmacokinet Biopharm. 1991;19(4):377-84. https://doi.org/10.1007/bf01061662.

12. Li ZL, Liu YX, Jiao Z, Qiu G, Huang JQ, Xiao YB, et al. Population pharmacokinetics of Vancomycin in Chinese ICU neonates: initial dosage recommendations. Front Pharmacol. 2018;9:603. https://doi.org/10.3389/fpha r.2018.00603.

13. Cockcroft DW, Gault MH. Prediction of Creatinine clearance from serum Creatinine. Nephron. 1976;16(1):31-41. https://doi.org/10.1159/000180580.

14. Purwonugroho TA, Chulavatnatol S, Preechagoon Y, Chindavijak B, Malathum K, Bunuparadah P. Population pharmacokinetics of vancomycin in Thai patients. ScientificWorldJournal. 2012;2012:762649-8. https://doi. org/10.1100/2012/762649.

15. Keizer RJ, Karlsson MO, Hooker A. Modeling and simulation workbench for NONMEM: tutorial on Pirana, PsN, and Xpose. CPT Pharmacometrics Syst Pharmacol. 2013;2(6):e50. https://doi.org/10.1038/psp.2013.24.

16. Deng C, Liu T, Wu K, Wang S, Li L, Lu H, et al. Predictive performance of reported population pharmacokinetic models of vancomycin in Chinese adult patients. J Clin Pharm Ther. 2013;38(6):480-9. https://doi.org/10.1111/ jcpt.12092. 
17. Balch AH, Constance JE, Thorell EA, Stockmann C, Korgenski EK, Campbell SC, et al. Pediatric vancomycin dosing: trends over time and the impact of therapeutic drug monitoring. J Clin Pharmacol. 2015;55(2):212-20. https:// doi.org/10.1002/jcph.402

18. Anderson BJ, Allegaert $\mathrm{K}$, Van den Anker $\mathrm{JN}$, Cossey V, Holford NH. Vancomycin pharmacokinetics in preterm neonates and the prediction of adult clearance. Br J Clin Pharmacol. 2007;63(1):75-84. https://doi.org/1 0.1111/j.1365-2125.2006.02725.x.

19. Sanchez JL, Dominguez AR, Lane JR, Anderson PO, Capparelli EV, CornejoBravo JM. Population pharmacokinetics of vancomycin in adult and geriatric patients: comparison of eleven approaches. Int J Clin Pharmacol Ther. 2010; 48(8):525-33. https://doi.org/10.5414/cpp48525.

20. de Gatta Garcia M d MF, Revilla N, Calvo MV, Dominguez-Gil A, Sanchez Navarro A. Pharmacokinetic/pharmacodynamic analysis of vancomycin in ICU patients. Intensive Care Med. 2007;33(2):279-85. https://doi.org/10.1007/ s00134-006-0470-5.

21. Revilla N, Martin-Suarez A, Perez MP, Gonzalez FM, Fernandez de Gatta Mdel M. Vancomycin dosing assessment in intensive care unit patients based on a population pharmacokinetic/pharmacodynamic simulation. $\mathrm{Br}$ J Clin Pharmacol. 2010;70(2):201-12. https://doi.org/10.1111/j.1365-2125.2010.03 679.x.

22. Llopis-Salvia P, Jimenez-Torres NV. Population pharmacokinetic parameters of vancomycin in critically ill patients. J Clin Pharm Ther. 2006;31(5):447-54. https://doi.org/10.1111/j.1365-2710.2006.00762.x.

23. Schleibinger M, Steinbach CL, Topper C, Kratzer A, Liebchen U, Kees F, et al. Protein binding characteristics and pharmacokinetics of ceftriaxone in intensive care unit patients. Br J Clin Pharmacol. 2015;80(3):525-33. https:// doi.org/10.1111/bcp.12636

24. Rybak M, Lomaestro B, Rotschafer JC, Moellering R Jr, Craig W, Billeter M, et al. Therapeutic monitoring of vancomycin in adult patients: a consensus review of the American Society of Health-System Pharmacists, the Infectious Diseases Society of America, and the Society of Infectious Diseases Pharmacists. Am J Health Syst Pharm. 2009;66(1):82-98. https://doi.org/1 0.2146/ajhp080434.

25. Gwee A, Cranswick N, McMullan B, Perkins E, Bolisetty S, Gardiner K, et al. Continuous Versus Intermittent Vancomycin Infusions in Infants: A Randomized Controlled Trial. Pediatrics. 2019;143(2). https://doi.org/10.1542/ peds.2018-2179

\section{Publisher's Note}

Springer Nature remains neutral with regard to jurisdictional claims in published maps and institutional affiliations.

Ready to submit your research? Choose BMC and benefit from:

- fast, convenient online submission

- thorough peer review by experienced researchers in your field

- rapid publication on acceptance

- support for research data, including large and complex data types

- gold Open Access which fosters wider collaboration and increased citations

- maximum visibility for your research: over $100 \mathrm{M}$ website views per year

At BMC, research is always in progress.

Learn more biomedcentral.com/submissions 